PESQUISA

\title{
EFETIVIDADE DO INCENTIVADOR MUSCULAR INSPIRATÓRIO NA FUNÇÃO PULMONAR EM PACIENTES LAPARATOMIZADOS
}

\author{
Southier PD*, Netto $\mathrm{LB}^{\dagger}$, Kroth $\mathrm{A}^{\ddagger}$
}

\begin{abstract}
Resumo
Alterações da função pulmonar após cirurgias abdominais como a laparotomia levam à redução do volume pulmonar, prejudicando as trocas gasosas. Este estudo teve-se como objetivo avaliar e comparar os efeitos do incentivador muscular inspiratório (POWERbreathe-K5) e de exercícios de padrões respiratórios sobre a função pulmonar e a força muscular respiratória em pacientes submetidos à laparotomia. Trata de um estudo clínico randomizado transversal composto por 14 indivíduos (idade: 52,1 士16,07), que foram submetidos à laparotomia, sete realizaram 10 sessões de tratamento com o incentivador muscular respiratório (POWERbreathe-K5), e sete realizaram 10 sessões de tratamento com fisioterapia

\footnotetext{
" Graduada no Curso de Fisioterapia pela Universidade do Oeste de Santa Catarina de Joaçaba; Rua Getúlio Vargas, 2125, Flor da Serra, 89600-000, Joaçaba, Santa Catarina, Brasil; pamesouthier@ hotmail.com

† Graduada no Curso de Fisioterapia pela Universidade do Oeste de Santa Catarina de Joaçaba; Rua Getúlio Vargas, 2125, Flor da Serra, 89600-000, Joaçaba, Santa Catarina, Brasil; larissa_botegal@ hotmail.com

¥ Mestre em Ciências Biológicas e Fisiologia pela Universidade Federal do Rio Grande Do Sul; Professora na Universidade do Oeste de Santa Catarina; Rua Getúlio Vargas, 2125, Flor da Serra, 89600000, Joaçaba, Santa Catarina, Brasil; adarly.kroth@unoesc.edu.br
} 
convencional. Foram avaliadas na primeira e decima sessão de tratamento, as pressões respiratórias máximas (PImáx e PEmáx), o S-index (índice de intensidade) e fluxo inspiratório (PIF) de todos os participantes. Verificou-se que os dois grupos estudados apresentaram resultados estaticamente significativos ao comparar valores de pré e pós-tratamento, porém ao comparar os resultados entre os dois grupos, não se obteve diferença significativa, o que pode ocorrer devido aos sintomas dos pacientes no pós-operatório imediato. Dessa forma, concluiu-se que ambos os protocolos de tratamento contribuíram para a recuperação dos pacientes, porém ao realizar treinamento muscular inspiratório utilizan do o dispositivo POWERbreathe-K5, mostrou-se melhoras significativas em alguns parâmetros e na recuperação precoce da função pulmonar e da força muscular dos pacientes submetidos à laparotomia.

Palavras-chave: Laparotomia. Exercícios respiratórios. Mecânica respiratória. Modalidades de Fisioterapia.

\section{Effectiveness of inspiratory muscle stimulator in lung function in patients submitted to}

\section{Laparotomy}

\section{Abstract}

Changes in pulmonary function after abdominal surgery such as laparotomy lead to lung volume reduction, compromising gas exchange. This study aimed to evaluate and compare the effects of the inspiratory muscle stimulator (POWERbreathe-K5) and breathing patterns exercises on the pulmonary function and the respiratory muscle strength in patients submitted to laparotomy. It is a randomized cross-sectional study composed of 14 individuals (age: $52.1 \pm 16.07$ ), who were submitted to laparotomy; seven performed 10 treatment sessions with the inspiratory muscle stimulator (POWERbreathe-K5), and seven performed 10 treatment sessions with conventional physiotherapy. On the first and tenth treatment sessions, the maximal respiratory pressures (MIP and MEP), the S-index (intensity index), and the Inspiratory Flow (PIF) of all the participants were evaluated. It was verified that both studied groups presented statistically significant results when pre and post-treatment values were compared, however, when the results between the two groups were compared, no significant difference was obtained, maybe because of the symptoms of the patients in the immediate postoperative period. Therefore, it was concluded that both treatment protocols contributed to the recovery of the patients, however, when performing inspiratory muscle training, the device POWERbreathe-K5 showed satisfactory improvement in some parameters and in the early recovery of lung function and muscle strength of patients submitted to laparotomy. 
Keywords: Laparotomy. Breathing exercises. Respiratory mechanics. Physical therapy modalities.

\section{INTRODUÇÃO}

Mesmo com o advento de novas tecnologias que visam melhorar a qualidade de vida e diminuir o número de complicações relacionadas à doença, muitas complicações relacionadas às doenças e seus tratamentos ainda estão presentes.' As complicações pós-cirúrgicas são frequentes e ocorrem devido à existência de fatores de risco pré-operatórios, como idade avançada, presença de doença pulmonar prévia ou outras doenças clínicas, tabagismo, obesidade, desnutrição, tempo de cirurgia e técnica cirúrgica. As cirurgias abdominais apresentam uma alta incidência de complicações pulmonares. Estima-se que haja redução da capacidade vital de 50 a 60\% após cirurgias de tórax e abdome, e 30\% da capacidade residual funcional.'

A Laparotomia é uma intervenção cirúrgica frequentemente realizada, na qual é feita uma incisão cirúrgica da cavidade abdominal, e tem como objetivo avaliar a extensão da patologia ou identificar outras possíveis patologias ou lesões na área. Ela pode ser elencada como eletiva, exploratória, ou como via de drenagem de coleções líquidas. As laparotomias podem ser classificadas com base nas suas diferentes características, quanto à relação da cicatriz, que pode ser supraumbilical, periumbilical ou infraumbilical, quanto à relação na linha média do abdômen, em medianas ou paramedianas, quanto à relação dos músculos, quanto à direção, podendo ser longitudinal, transversal, oblíqua ou combinada, ou quanto à complexidade, sendo simples, com somente uma incisão, ou combinada, com associação de incisões. ${ }^{2}$

No pós-operatório de laparotomia, o paciente pode apresentar diferentes complicações, como alterações nos órgãos que receberam algum tipo de intervenção, em razão da anestesia e da cicatrização. Geralmente, as complicações mais frequentes são infecção, fístula anastomótica ou obstrução intestinal, sepse, infecções urinárias ou complicações respiratórias, ${ }^{3}$ que podem alterar a mecânica respiratória, as trocas gasosas e o volume pulmonar. Estas complicações aumentam o período de internação do paciente, elevando os gastos e aumentando a mortalidade. As prevalências de complicações respiratórias no pós-operatório variam de 17 a $88 \%{ }^{4}$

Durante o ciclo respiratório a inspiração de indivíduos saudáveis faz com que o conteúdo abdominal se mova anteriormente em decorrência da contração do diafragma, aumentando a pressão abdominal e permitindo melhor expansibilidade torácica. Em razão de um procedimento cirúrgico, o conteúdo abdominal sofre deslocamento, que altera a mecânica respiratória e, consequentemente, ocasiona 
a redução da capacidade vital, pela diminuição da força diafragmática que acarreta a diminuição dos volumes pulmonares, levando a um quadro de hipoxemia e, possivelmente, à ocorrência de uma atelectasia. ${ }^{5}$ Além disso, o procedimento causa alterações nas trocas gasosas e na complacência torácica e pulmonar, sendo comum que o paciente desenvolva um padrão restritivo de disfunção pulmonar, que está relacionado à diminuição do movimento do diafragma. Essas complicações podem ser evitadas ou minimizadas pelo atendimento de fisioterapia respiratória, da qual um dos objetivos principais é a melhora da força muscular respiratória. ${ }^{6}$

A fisioterapia respiratória é uma das medidas empregadas visando diminuir as complicações da perda funcional pulmonar. Para isso, são utilizadas várias técnicas de expansão pulmonar, reeducação respiratória, cinesioterapia respiratória, entre outras inúmeras técnicas que podem auxiliar na reabilitação da capacidade respiratória. ${ }^{7}$ A realização de fisioterapia no pós-operatório de laparotomia pode prevenir ou minimizar o aparecimento de complicações. Além disso, uma avaliação criteriosa de cada paciente pode identificar anormalidades no período pré-operatório e direcionar os cuidados conforme as condições dos pacientes, podendo, também, reduzir as complicações pulmonares pós-operatórias. ${ }^{8}$

O Incentivador Muscular inspiratório (POWERbreathe) é um dispositivo de treinamento da musculatura inspiratória, incentivador respiratório, que auxilia na melhora da força dos músculos responsáveis pela respiração. ${ }^{9}$ Destinado ao treinamento dos músculos inspiratórios, o POWERbreathe é um dispositivo que aumenta a resistência respiratória e a força muscular respiratória, diminuindo a fadiga muscular e a falta de ar, sendo útil também para controle médico. Estudos ${ }^{10}$ concluíram que o treinamento muscular inspiratório pode reduzir complicações pulmonares pós-operatórias e diminuir o tempo de internação do paciente.

Ao realizar o treinamento com o POWERbreathe, é possível sentir uma resistência ao realizar a inspiração, essa resistência é introduzida gradualmente nas cinco primeiras respirações de uma sessão de treinamento. Durante cada respiração é possível notar que a resistência é máxima no início da inspiração e é suavizada gradualmente conforme os pulmões vão se enchendo de ar. Esse é o efeito de carga do POWERbreathe, que é variável e alternada durante a respiração para ser correspondente à mudança das forças musculares do músculo respiratório. É recomendado que a rotina de treinamento consista em 60 respirações, duas vezes por dia, totalizando 5 a 10 minutos de treinamento por dia. Para iniciar o treinamento o paciente deve se posicionar em pé ou sentado, segurando o dispositivo na parte inferior, para não cobrir a entrada de ar. Depois de posicionado corretamente, o paciente deve inspirar o mais forte, rápido e profundamente que conseguir, pois é na inspiração que ocorre o treinamento. Após, o paciente deve expirar de forma lenta e passiva pela boca, até que os pulmões estejam completamente vazios, 
fazendo com que os músculos do tórax e ombros relaxem. É recomendado o uso do clipe nasal para ajudar a respirar somente pela boca. ${ }^{11}$

O dispositivo utilizado para o treinamento envia os resultados obtidos pelos indivíduos para o computador, no qual, a partir de um software específico do POWERbreathe-K5, esses resultados são possíveis serem visualizados, tanto na forma numérica quanto por gráficos, trazendo vários resultados, sendo os principais deles o S-index, que é o índice de intensidade em que o paciente realiza a respiração, e a PIF, que é fluxo inspiratório de cada ciclo respiratório."

Esse dispositivo foi inventado por Alison McConnell, na Espanha, porém saiu para o mercado para uso somente em 1997. Desde 2011 cresceu muito o atendimento no mundo, os distribuidores internacionais promoveram reuniões nas quais foram realizadas demonstrações, expandindo seu uso para países como Austrália, Japão, Coréia, Rússia e Brasil. ${ }^{12}$ Ainda é pouco conhecido, sendo utilizado com mais frequência na área desportiva; há poucas pesquisas com o aparelho, ${ }^{13}$ diante disso, neste estudo teve-se como objetivo verificar a eficácia do incentivador muscular inspiratório em pacientes submetidos à técnica de laparotomia, além de avaliar o perfil sócio demográfico dos pacientes, avaliar a efetividade dos protocolos na função pulmonar dos pacientes que receberam tratamento e avaliar qual dos protocolos apresentou melhor resposta terapêutica nos pacientes atendidos.

\section{MATERIAL E MÉTODOS}

Esta pesquisa consiste em um estudo clínico randomizado transversal. Primeiramente, foi realizado o contato com a instituição, para que o tema fosse explicado aos responsáveis e para ser questionado sobre a viabilidade do estudo. Após a aprovação da instituição, o trabalho foi enviado ao CEP/Hust, onde foi aprovado com o Parecer n. 1.576.392. A amostra foi composta por 14 indivíduos hospitalizados, submetidos a algum procedimento cirúrgico de laparotomia, com ressecção mediana, em um Hospital Universitário do Meio-Oeste de Santa Catarina. Todos os participantes e/ou acompanhantes receberam um Termo de Consentimento Livre Esclarecido (TCLE), bem como assinaram uma autorização para divulgação de dados.

Os critérios de inclusão do estudo foram a permanência mínima de cinco dias na unidade hospitalar, pacientes lúcidos, colaborativos e ativos, submetidos ao procedimento de laparotomia com ressecção mediana. Foram excluídos do estudo os pacientes que apresentaram confusão mental, sedados, com obesidade mórbida, com fraturas de face, traqueostomia, e pacientes em ventilação mecânica ou que durante o tratamento tiveram alta hospitalar.

A coleta de dados foi realizada nos meses de junho a agosto de 2016. Após aceitação dos pacientes ou acompanhantes, estes responderam um questionário 
estruturado com 10 perguntas, sendo cinco abertas e cinco fechadas, as quais contemplavam os dados do paciente, o motivo de internação, as comorbidades, se era fumante ou etilista, em caso positivo, se parou e há quanto tempo, e outras anotações referentes ao paciente que fossem encontradas descritas em prontuário.

\subsection{PROTOCOLO DE TRATAMENTO}

O protocolo de tratamento consistia em separar os pacientes de forma aleatória em dois grupos; o primeiro paciente foi sorteado para qual grupo pertenceria, e os demais foram alocados aleatoriamente em um dos dois grupos. Todos os pacientes iniciaram o protocolo de atendimento em até 24 horas após o procedimento cirúrgico.

O grupo de estudo (GE) foi composto por sete indivíduos os quais utilizaram o incentivador muscular inspiratório (POWERbreathe-K5), realizando uma série de 30 repetições por sessão. Foi realizada também a cinesioterapia motora, com alongamentos passivos e ativos mantidos por 30 segundos, três séries de 10 repetições para cada exercício de fortalecimento realizado, além de deambulação nos pacientes que conseguiam realizar. Após a realização do atendimento os pacientes foram posicionados e acomodados no leito pelos aplicadores. Foram realizadas 10 sessões (duas sessões diárias por cinco dias consecutivos).

O grupo controle (GC) foi composto por sete indivíduos, os quais realizaram fisioterapia convencional utilizando padrões respiratórios como: inspiração fragmentada, soluços inspiratórios, freno labial, expiração abreviada e inspiração máxima sustentada, uma sessão de 10 repetições por exercício. Foi realizada, também, a cinesioterapia motora, com alongamentos passivos e ativos mantidos por 30 segundos, três séries de 10 repetições para cada exercício de fortalecimento realizado, além de deambulação nos pacientes que conseguiam realizar. Após a realização do atendimento os pacientes foram posicionados e acomodados no leito pelos aplicadores. Foram realizadas 10 sessões (duas sessões diárias por cinco dias consecutivos).

Após ser definido o método de tratamento foi realizada avaliação física, contendo ausculta pulmonar, pressão inspiratória máxima (PImáx), pressão expiratória máxima (PEmáx) verificado pelo Manovacuômetro (GERAR), S-index e Pico de fluxo inspiratório (Software Powerbreathe-K5), além de verificação quanto à localização, extensão e aspecto da cicatriz cirúrgica. Essa avaliação física foi realizada na primeira e na décima sessão.

A análise estatística dos dados foi realizada de forma inferencial por meio da apresentação dos dados em tabelas e gráficos. Os dados foram avaliados utilizando-se um Teste † e Análise de variância (ANOVA - uma via), para se avaliar a eficácia do treinamento dos músculos inspiratórios em pacientes submetidos 
à cirurgia de laparotomia mediana. Em todas as análises foi utilizado o nível de significância de 95\% ( $\mathrm{p}<0,05)$. Utilizou-se o programa software Graph Prism 5.0 e o teste post hoc de comparações múltiplas de Dunnett's sempre que apropriado (ANOVA) e Mann Whitney teste (Teste †).

\section{RESULTADOS}

A população foi composta por 14 pacientes, sendo 10 indivíduos do sexo masculino (70\%) e quatro do sexo feminino (30\%); a média geral de idade foi de 52,1 anos (+16,07); destes, 60\% eram fumantes, com uma média de 11 anos de consumo de cigarro, $20 \%$ eram etilistas e $7 \%$ eram usuários de drogas. As causas para a realização da laparotomia foram neoplasias e acidentes automobilísticos.

O grupo de estudo (GE) foi composto por sete indivíduos, sendo seis do sexo masculino e um do sexo feminino, com média de idade de 47,8 anos $(+19,65)$; foi constatado que $43 \%$ destes eram fumantes, somente um indivíduo era etilista (14\%) e um era usuário de drogas (14\%), nenhum deles apresentava comorbidade associada. O grupo controle (GC) foi composto por sete indivíduos, sendo quatro do sexo masculino e três do sexo feminino, com média de idade foi 56,4 anos $(+11,43)$; verificou-se que $71 \%$ destes eram fumantes, $29 \%$ eram etilistas; nenhum indivíduo era usuário de drogas, quatro eram hipertensos e um diabético.

Os valores referentes a PImáx e PEmáx após 10 sessões em ambos os grupos que receberam diferentes protocolos de tratamento, não se observou diferença significativa $(p=0,2656)$ (Tabela 1$)$.

Tabela 1 - Comparação de PImáx e PEmáx entre GE e GC pré e pós 10 sessões de treinamento muscular inspiratório§

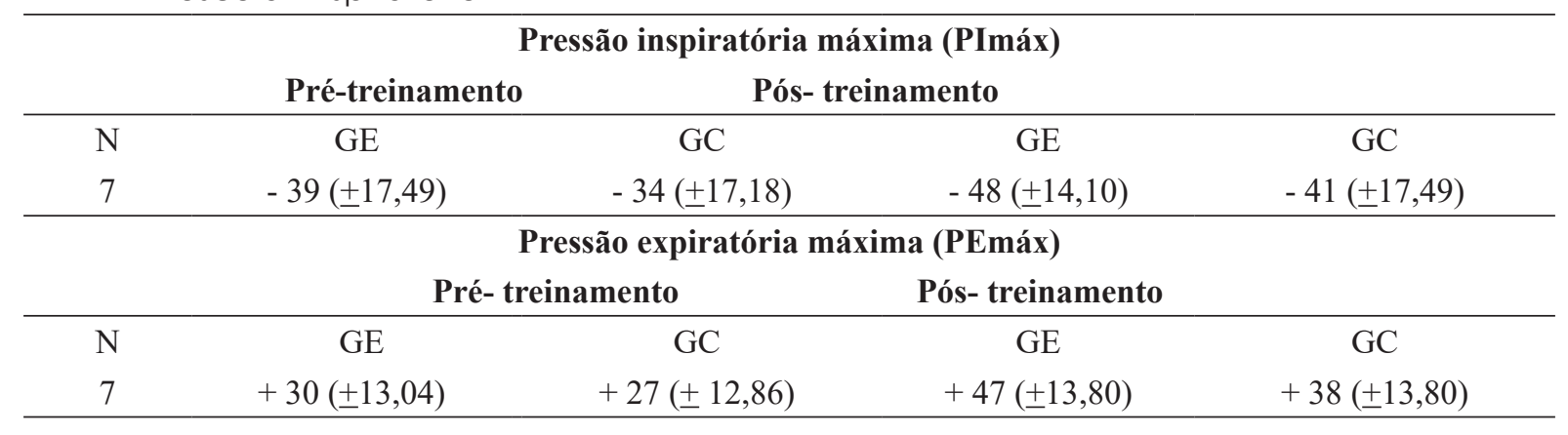

Ao se avaliar o índice de intensidade S-index no grupo GE verificou-se que houve diferença significativa após 10 sessões de utilização do incentivador inspiratório (POWERbreathe-K5) $(\mathrm{p}<0,01)$ (Figura 1A). Porém, no grupo GC não

$\S \mathrm{N}=$ número de indivíduos; GE = Grupo de estudo; GC = Grupo controle. Teste $t$ teste post hoc Mann Whitney teste. 
houve diferença significativa entre o S-index após as 10 sessões de fisioterapia convencional $(p=0,68)$ (Figura 1B).
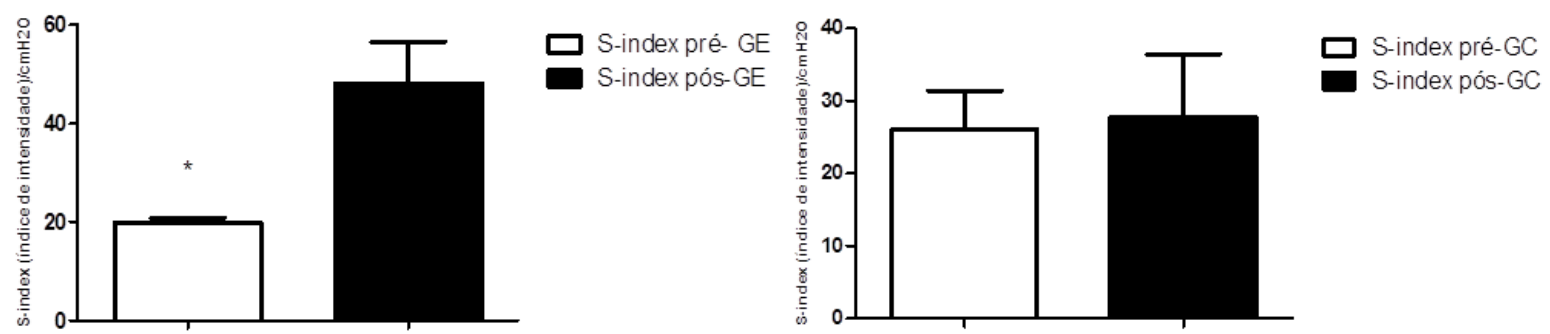

Figura 1 - Índice de intensidade (S-index) pré e pós 10 sessões de treinamento muscular inspiratório".

Ao analisar os valores do fluxo inspiratório (PIF) comparando os valores de pré e pós 10 sessões de tratamento, verificou-se que houve diferença significativa no grupo GE ( $p<0,0084)$ (Figura 2A), o que não ocorreu no grupo $G C(p=0,8712)$ (Figura 2B).
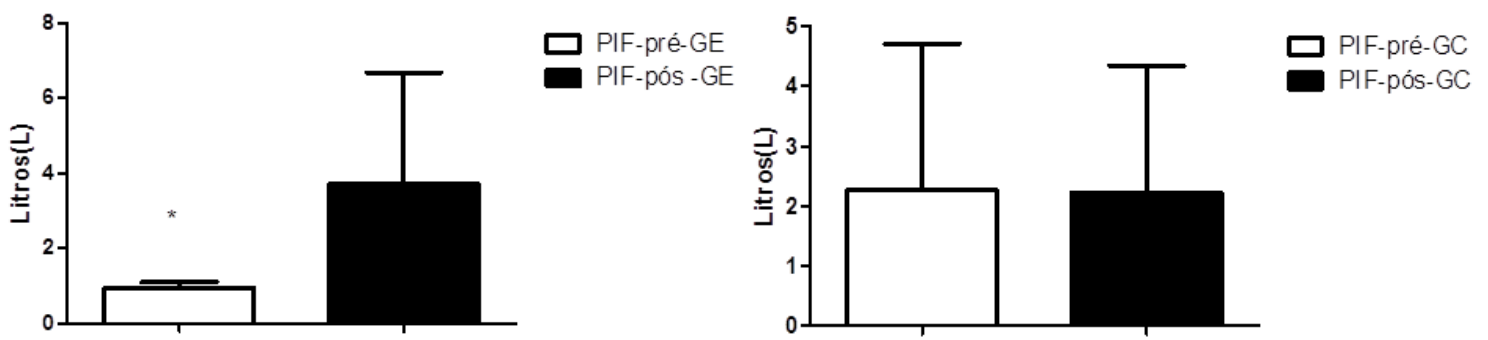

Figura 2 - Fluxo inspiratório (PIF) pré e pós 10 sessões de treinamento muscular inspiratórioll.

Através da análise da variância (ANOVA-Uma via) quando foram comparados os dois protocolos de tratamento após 10 sessões, verificou-se que não houve diferença significativa entre os grupos em relação Pimax $\left(F_{0,8213}=0,09311, p=0.95\right)$, Pemax $\left(F_{2,601}=0,2618, p=0,99\right)$, S-index $\left(F_{7,299}=0,5105, p=0,06\right)$ e PIF $\left(F_{1,745}=0,1791\right.$, $p=0,1846)$. Esse resultado pode ter ocorrido em razão do número de participantes, do curto período de tratamento ou das inúmeras complicações pós-operatórias que ocorreram em alguns pacientes, como dor, quadros de náusea e vômito ou ao uso de sonda nasogástrica e drenos.

\section{DISCUSSÃO}

A laparotomia tem por principal objetivo ter acesso aos órgãos intraabdominais a partir de uma secção na região abdominal, permitindo a exploração desses órgãos. Esse procedimento causa grande exposição anatômica, além

\footnotetext{
"Valores de * $p<0,05$ quando comparado pré e pós 10 sessões de treinamento muscular inspiratório. Os resultados foram expressos na forma de média \pm DP. N=7. Teste † teste post hoc Mann Whitney teste.
} 
disso, leva a possíveis complicações na mecânica e no padrão respiratório em razão do acometimento dos músculos respiratórios, assim, os autores relatam em seu estudo, que a fisioterapia é de suma importância no período pós-operatório destes pacientes, trazendo ótimos resultados na reabilitação e evitando outras complicações que podem surgir. ${ }^{3}$

Neste estudo verificou-se que houve predomínio de indivíduos do sexo masculino entre os participantes, com média de idade de 52 anos, o que corrobora outro estudo pesquisado, ${ }^{15}$ no qual os autores buscaram analisar a morbidade e a mortalidade de 4.346 pacientes que necessitaram de laparotomia de emergência e obtiveram a mesma prevalência de sexo e a mesma faixa etária.

Os motivos para realizar esse procedimento cirúrgico são variados, porém as causas mais frequentes neste estudo foram neoplasias (80\%) e acidentes automobilísticos (20\%). O que vai de encontro ao estudo do realizado por Mentula e Leppäniemi, ${ }^{16}$ que trazem as patologias oncológicas como principal causa para realização de laparotomia. Outro estudo realizado ${ }^{4}$ demonstrou que o diagnóstico de tumores gastrointestinais e a realização de cirurgia abdominal ocorriam em maior incidência em pacientes do sexo masculino (66\%), o que corrobora este estudo.

Em pesquisa sobre o treinamento muscular no período pós-operatório em pacientes submetidos à cirurgia de revascularização do miocárdio, 95\% da população da sua amostra eram hipertensos, e $60 \%$ eram diabéticos, ${ }^{17}$ o que coincide com a amostra deste estudo, no qual as principais comorbidades relatadas foram a hipertensão arterial e a diabetes. Os hábitos de vida mais observados foram o tabagismo e o etilismo, o que vai ao encontro da pesquisa citada, da qual $40 \%$ dos pacientes eram tabagistas e $10 \%$ etilistas. Essas comorbidades e esses hábitos de vida são considerados um grande fator de risco para desenvolver outras doenças associadas a problemas cardíacos e respiratórios, estando inteiramente ligados ao processo de recuperação dos pacientes. Não foi encontrado na literatura nenhum estudo realizado com pacientes laparatomizados, que traga as comorbidades encontradas em nosso estudo.

A manovacuometria tem como objetivo mensurar a força da musculatura inspiratória e expiratória, determinada pela pressão negativa e pela pressão positiva que é exercida pelo dispositivo no qual o paciente realiza inspiração e expiração rápida e profunda em seu bocal. Em um estudo realizado em 2010 foram avaliadas a Pimax e a Pemax de 140 indivíduos saudáveis, para então se chegar a um parâmetro de valores-referência para eles. No estudo foi verificado que a Pimax ideal em indivíduos saudáveis na faixa etária de 50 anos (faixa etária deste estudo) era de $-115 \mathrm{cmH}_{2} \mathrm{O}$ em homens, e $-85 \mathrm{cmH}_{2} \mathrm{O}$ em mulheres, já a Pemax

\footnotetext{
$\overline{\| V a l o r e s ~ d e ~}{ }^{*} p<0,05$ quando comparado pré e pós 10 sessões de treinamento muscular inspiratório. Os resultados foram expressos na forma de média \pm DP. N=7. Teste t teste post hoc Mann Whitney teste.
} 
ideal seria de $+109 \mathrm{cmH}_{2} \mathrm{O}$ em homens e $+95 \mathrm{cmH}_{2} \mathrm{O}$ em mulheres. ${ }^{20}$ Porém, no presente estudo, não se alcançou esse valor de referência em nenhum paciente, possivelmente pelo fato de eles estarem em um quadro patológico e terem sido submetidos a um procedimento cirúrgico.

Em outro estudo no qual foi avaliada a força muscular inspiratória e expiratória em idosas praticantes de atividade física e sedentárias, foi relatado que uma Pimax abaixo de $-80 \mathrm{cmH}_{2} \mathrm{O}$ e uma Pemax superior a $+90 \mathrm{cmH}_{2} \mathrm{O}$ afasta indícios de fraqueza muscular. ${ }^{21}$ Neste estudo foi também relatado que os valores-referência de fraqueza muscular apresentam uma Pimax entre - 70 e $-46 \mathrm{cmH}_{2} \mathrm{O}$; em pacientes com fadiga muscular respiratória a Pimax seria entre -44 e $-25 \mathrm{cmH}_{2} \mathrm{O}$, e pacientes com falência muscular respiratória apresentariam Pimax igual a $-20 \mathrm{cmH}_{2} \mathrm{O}$. O que vai ao encontro dos dados da presente amostra, pois fica evidente que tanto o grupo GE quanto o grupo GC se encontravam em um estado de falência muscular respiratória ao ser avaliada a Pimax no período de pré tratamento. Isso mostra um ganho significativo em ambos os grupos no período pós-treinamento, visto que os pacientes passaram de um estado de fadiga da musculatura respiratória para fraqueza muscular respiratória.

As cirurgias abdominais como a laparotomia trazem como principal sintoma a dor, que está presente em grande parte dos pacientes e pode resultar em sofrimento e exposição destes a riscos desnecessários no pós-operatório. O controle da dor nesta etapa envolve basicamente 0 uso de analgésicos e anti-inflamatórios. ${ }^{14}$ No presente estudo observou-se que a dor é constante no período pós-operatório imediato, e que fazer uso da medicação traz uma melhora significativa em relação aos atendimentos de fisioterapia; porém, mesmo com o uso das medicações, a dor se demonstrou como um possível motivo para a baixa significância de resultados de manovacuometria, considerando que a dor leva a uma diminuição da força muscular; dessa forma, pode ter sido a presença de dor no dia da avaliação póstratamento que impossibilitou resultados melhores.

Os grupos foram considerados homogêneos em relação às variáveis, em que se observou que houve melhora na capacidade respiratória dos pacientes, principalmente no grupo em que foi realizado o incentivar muscular inspiratório (POWERbreathe-K5); porém, ao comparar os valores de Pimax e Pemax entre os grupos, não houve diferenças significativas entre os tratamentos, o que pode ter ocorrido em razão do quadro álgico, episódios de náusea e vômitos que eram frequentes na maioria pacientes, que acarretou no quadro de enfraquecimento global. A maioria dos pacientes fazia uso de sonda nasogástrica e drenos, o que dificultava mais a realização do tratamento, além disso, todos os pacientes eram reavaliados ao final de cada sessão, e muitos relatavam estar cansados, o que pode ter determinado a baixa significância de resultados. 
Também foi verificada a efetividade do treinamento muscular em pacientes no pós-operatório de cirurgias de revascularização do miocárdio, divididos em um grupo controle e em outro grupo que realizou treinamento muscular com 0 dispositivo Threshold, o qual a partir de uma válvula fechada com pressão positiva proporciona uma carga durante o ciclo respiratório, fazendo assim o mesmo fortalecimento dos músculos respiratórios que o dispositivo utilizado neste estudo.17 Os autores utilizaram a Manovacuometria como um dos parâmetros analisados, o que corrobora o presente estudo, tendo como resultados semelhantes de Pimax e Pemax ao serem comparados ambos os grupos. ${ }^{17}$

A medida de S-index permite identificar se há ou não fraqueza funcional, e apesar de não haver consenso sobre o valor de referência, refere-se que um S-index de $80 \mathrm{cmH} 2 \mathrm{O}$ é um valor considerável e que pode ser usado como parâmetro. 18 Neste estudo, ao comparar os valores de S-index no pré e pós-operatório do GE, foi verificada significante melhora, o que possivelmente aconteceu em razão do fortalecimento muscular que ocorreu no decorrer dos treinamentos, visto que ao longo dos dias os pacientes demonstravam maior facilidade para realizar os 30 ciclos do treinamento. Porém, no GC, os valores de S-index não apresentaram valores significativos comparando-se o pré e o pós-tratamento, isso ocorreu em razão da baixa intensidade de fortalecimento dos músculos ao realizarem os exercícios de padrões respiratórios.

Quanto aos valores de PIF, não existem estudos na literatura que tragam valores de referência, sendo verificado no presente estudo, ao comparar os valores de pré e pós 10 sessões de tratamento, diferenças significativas no grupo GE, possivelmente em decorrência do fortalecimento muscular após os treinamentos, porém no grupo GC não houve diferenças significativas pela baixa intensidade dos exercícios de padrões respiratórios.

Os resultados de S-index e PIF obtidos no grupo GE deste estudo corroboram com uma pesquisa na qual os autores realizaram um estudo com 19 indivíduos saudáveis, um treino dos músculos inspiratórios, sendo dividida a população em dois grupos, um que realizou um treinamento com o dispositivo POWERbreathe Classic $\AA^{\circledR}$ Level 1 e Level 2, e um que não realizou nenhuma intervenção.19 Ao compararmos os dois grupos em relação a esses parâmetros não se observaram diferenças significativas.

É necessário ressaltar que não foram encontrados na literatura trabalhos associando o uso do incentivador muscular inspiratório em pacientes submetidos a procedimentos cirúrgicos, e que são poucos os estudos que relacionam as complicações respiratórias com as cirurgias abdominais como a laparotomia, o que sugere a importância e o pioneirismo do presente estudo. 


\section{CONSIDERAÇÕES FINAIS}

De acordo com os resultados deste estudo, concluiu-se que o treinamento muscular inspiratório com o dispositivo POWERbreathe-K5 contribuiu para a recuperação da função pulmonar e força muscular dos pacientes submetidos à laparotomia, bem como que o dispositivo pode ser indicado no tratamento ou prevenção de complicações pulmonares pós-operatórias. Além disso, o estudo contribuiu para a inserção do POWERbreathe-K5 no ambiente hospitalar. Diante disso, fazem-se necessários mais estudos sobre os benefícios que pode trazer o POWERbreathe-K5 em pacientes com complicações do trato respiratório.

\section{REFERÊNCIAS}

1. Joia Neto L, Thomson JC, Cardoso JR. Complicações respiratórias no pósoperatório de cirurgias eletivas e de urgência e emergência em um Hospital Universitário. J. bras. pneumol. 2005: 41-7.

2. Tazima MFGS, Vicente YAMVA, Moriya T. Laparotomia. Medicina. 201 1; 44(1): 33-8.

3. Silva FS, Lopes TM, Duarte J, Medeiros RF. Tratamento fisioterapêutico no pósoperatório de laparotomia. J Health Sci Inst. 2010; 28(4):341-4.

4. Dias CM, Plácido TR, Ferreira MFB, Guimarães FS, Menezes SLS. Inspirometria de incentivo e breath stacking: repercussões sobre a capacidade inspiratória em indivíduos submetidos à cirurgia abdominal. Rev Bras Fisioter. 2008 mar-abr; 12(2):94-9.

5. Florencio R, Fregonezi G, Brilhante S, Silva AB, Dias F, Resqueti V. Heart Rate Variability at rest and after the 6-minute walk test (6MWT) in children with cystic fibrosis. Braz. J. Phys. Ther. 2013; 17(5):419-425.

6. Caruso P, Albuquerque ALP, Santana PV, Cardenas LZ, Ferreira JG, Prina E et al. Diagnostic methods to assess inspiratory and expiratory muscle strength. J. bras. pneumol. 2015 mar-abr; 41 (2). 
7. Gastaldi AC, Magalhães CMB, Barauna MA, Silva EMC, Souza HCD. Benefícios da cinesioterapia respiratória no pós-operatório de colecistectomia laparoscópica. Rev Bras Fisioter. 2008 mar-abr; 12(2):100-6.

8. Forgiarini Junior LAF, Carvalho AT, Ferreira TS, Monteiro MB, Bosco AD, Gonçalves MP et al. Atendimento fisioterapêutico no pós-operatório imediato de pacientes submetidos à cirurgia abdominal. J. bras. pneumol. 2009 maio;35(5):445-59 .

9. Charususin N, Gosselink R, Decramer M, Mcconnell A, Saey D, Maltais F et al. Inspiratory muscle training protocol for patients with chronic obstructive pulmonary disease (IMTCO study): a multicentre randomised controlled trial. BMJ Open. 2013:2-8.

10. Minahan C, Sheehan B, Doutreband R, Kirkwood T, Reeves D, Cross T. Repeated-Sprint Cycling Does Not Induce Respiratory Muscle Fatigue in Active Adults: Measurements from The Powerbreathe® Inspiratory Muscle Trainer. J Sports Sci Med. 2015:234-8.

11. Powerbreathe Ironman. Manual do Usuário [internet]. Inglaterra. 2011.

12. Powerbreathe Brasil. Site Oficial [internet]. 2015.

13. Hart N, Sylvester K, Ward S, Cramer D, Moxham K. Evaluation of an inspiratory muscle trainer in healthy humans. Respir Med. 2001 Jun; 95(6):526-31.

14. Miranda AS, Novaes RD, Ferreira AE, Neves MPC, Corréa CL, Mendonça VA. Avaliação da força da musculatura respiratória, pico de fluxo expiratório e dor após colecistectómia aberta. Acta Gastroenterol Latinoam. 2009; 39(1):38-46.

15. Tolstrup MB, Watt SK, Gogenur I. Morbidity and mortality rates after emergency abdominal surgery: an analysis of 4346 patients scheduled for emergency 
laparotomy or laparoscopy. Langenbecks Arch Surg. 2016 Aug:2-9.

16. Mentula PJ, Leppäniemi AK. Applicability of the Clavien-Dindo classification to emergency surgical procedures: a retrospective cohort study on 444 consecutive patients. Patient Saf Surg. 2014 Jul 26:8-31.

17. Matheus GB, Dragosavac D, Trevisan P, Costa CE, Lopes ML, Ribeiro GCAR. Treinamento muscular melhora o volume corrente e a capacidade vital no pósoperatório de revascularização do miocárdio. Rev Bras Cir Cardiovasc. 2012; 27(3):362-7.

18. Sclauser PIM, Franco PV, Fregonezi GA, Sheel AW, Chung F, Reid WD. Reference values for maximal inspiratory pressure: a systematic review. Can Respir J. 2014; 21 (1):43-50.

19. Esteves F, Santos I, Valeriano J, Tomás MT. Treino de músculos inspiratórios em indivíduos saudáveis: estudo randomizado controlado. Rev saúde e tecnol. 2016; (15):7-10.

20. Simões RP, Deus APL, Auad MA, Dionísio J, Mazzonetto M, Silva AB. Maximal respiratory pressure in healthy 20 to 89 year-old sedentary individuals of central São Paulo State. Revista Brasileira de Fisioterapia. 2010; 14(1):60-7.

21. Gonçalves MP, Tomaz CAB, Cassiminho ALF, Dutra MF. Avaliação da força muscular inspiratória e expiratória em idosas praticantes de atividade física e sedentárias. Rev. bras. ciênc. mov. 2006; 14(1):37-44.

Data de submissão: 03 de outubro de 2017 Avaliado em: 29 de abril de 2018 (AVALIADOR B) Avaliado em: 29 de abril de 2018 (AVALIADOR C) Avaliado em: 03 de maio de 2018 (AVALIADOR D) Aceito em: 16 de maio de 2018 